\title{
Ueber den Einfluß der Kriegskost auf die Ernährung, insbesondere der Jugend.
}

Von Prof. Dr. med. et phil. H. Griesbach in Straßburg.

Es fragt sich, ob die im Verlaufe des Krieges eingetretene Verminderung unserer Lebensmittel zu Befürchtungen Anlaß gibt, daß besonders die Jugend bei der jetzigen Kost in ihrer Entwicklung und Gesundheit geschädigt wird. An verschiedenen Orten des Reiches sind hierüber ärztlichen Untersuchungen angestellt worden und zum Teil noch im Gange. Veröffentlichungen aus den Jahren 1915 und 1916 von A. Kettner (Charlottenburg)' $)$, F. Lommel (Jena) ${ }^{2}$ ), und R. Hess (Straßburg) berichten, daß bei Säuglingen und Kindern im Spielalter ein nennenswerter Unterschied im Ernährungszustande im Vergleich zu Friedenszeiten nicht eingetreten sei. Nach Fehling) war das Durchschnittsgewicht von 300 Neugeborenen aus den Jahren 1914 und 1916 fast das gleiche. Im Jahre 1916 geborene Knaben waren um 1,2 cm, Mädchen um $0,4 \mathrm{~cm}$ kleiner als die von 1914. Kettner und die Mannheimer Schulärztin Frau Dr. Grätzer-Hepner ${ }^{5}$ ) haben bei Schulkindern und Lommel hat bei 14- bis 18jährigen Lehrlingen der Firma Zeiß zwar manchmal ein Zurückbleiben des Körpergewichtes im Verlaufe der Kriegszeit gefunden, eine Schädigung der Untersuchten war aber infolge der veränderten Ernährung ebenfalls nicht nachzuweisen. Nach A. Thiele (Chemnitz) ${ }^{6}$ ) hatten gesunde Knaben von 14 Jahren vor dem Kriege eine Körperlänge von $143 \mathrm{~cm}$ und ein Gewicht von $34,5 \mathrm{~kg}$. Im Jahre 1916 ergab die Untersuchung gleichaltriger Knaben eine Länge von

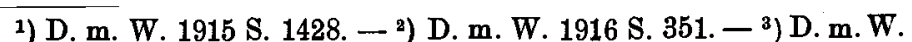
1916 S. 1620 - 4) D. m. W. 1917 S. 96. - i) Zschr. f. Schulgesdhtspfl. 1915 S. $545 .-6$ ) D. m. W. 1916 Nr. 28. 
$145,4 \mathrm{~cm}$ und ein Gewicht von $36,8 \mathrm{~kg}$. Von mangelhafter Ernährung während des Krieges war nichts zu merken. E. Engelh or n (Göppingen)1) gibt an, daß der Ernährungszustand von 4000 Schulkindern im Alter von 6 bis 13 Jahren während des Krieges durchschnittlich besser war als bei gleichaltrigen Kindern vor dem Kriege. Dieser Befund stützt sich fast ausschließlich auf den Gesamteindruck, den die Kinder machten, unter besonderer Berücksichtigung der Beschaffenheit des Fettpolsters, der Straffheit von Haut und Muskeln sowie der Farbe und Blutfülle der Schleimhäute. In einzelnen Fällen wurde auch das Körpergewicht ermittelt. Engelhorn möchte den günstigen Ernährungszustand der Kinder aus Landgemeinden dem reichlichen Genuß von Obst und Kriegsbrot zuschreiben. Dabei dürfte es sich dann aber wohl nicht nur um die Anregung der Verdauungstätigkeit durch Säuren handeln, wie Engel. horn meint, sondern auch um vermehrte Zufuhr von Vitaminen im Sinne C. Funks, F. Röhmanns Eiweißergänzungsstoffen. Nach Mitteilungen $\mathrm{D}$ i e ud onnés in der bayerischen Kammer der Abgeordneten²) ist eine Unterernährung bei Kindern bisher nicht eingetreten.

Vor kurzem hat E. Schlesing èr (Straßburg) ${ }^{3}$ ) Beobachtungen über den Einfluß der durch die Kriegslage veränderten Ernährung auf Schüler und Fortbildungsschulen besuchende Lehrlinge angestellt und die Ergebnisse mit denjenigen verglichen, die er bei Untersuchungen vor dem Kriege an gleichaltrigen und gleich gut entwickelten Angehörigen derselben Schulen erhielt. Es wurden sowohl Längen- als auch Gewichtsmessungen ausgeführt. Besonders interessant sind die vierteljährlich wiederholten Wägungen. 'Im ersten Vierteljahr 1916 zeigten $45 \%$ der Schüler Gewichtszunahme, $36 \%$ keinen wesentlichen Unterschied des Gewichts, $19 \%$ Gewichtsabnahme von $1 / 2-2 \mathrm{~kg}$. Nach Wägungen an derselben Schule im gleichen Zeitabschnitt vor dem Kriege betrug die Zahl der Knaben mit Gewichtszunahme 53\%, unbedeutende Veränderungen zeigten $35 \%$, Abnahme des Gewichts um $1 / 2-11 / 2 \mathrm{~kg}$ fand sich bei $12 \%$. Im zweiten Vierteljahr 1916 stieg bei denselben Schülern wie im ersten Vierteljahre die Zahl derjenigen mit Gewichtsabnahme von 19 auf $31 \%$, während sich die Zahl der Knaben mit Gewichtszunahme von 45 auf $26 \%$ verminderte. Vor dem Kriege betrug die Zahl im zweiten Vierteljahre der Schüler mit Gewichtsabnahme nicht mehr als $20 \%$, die der Schüler mit Gewichts: zunahme noch 51\%. Im dritten Vierteljahr 1916 ergaben die Wägungen, ebenso wie im gleichen Zeitraume vor dem Kriege, ganz andere Verhältnisse zwischen $\mathrm{Zu}$ - und Abnahme des Gewichtes. Bei $2 \%$ der Schüler, die im zweiten Vierteljahre eine Zunahme aufwiesen, hatte das Gewicht abgenommen, bei $7 \%$ blieb es unverändert, $90 \%$ wiesen eine $\mathrm{Zu}$ nahme auf. Dieses Ergebnis läßt nach Schlesinger auf einen Ausgleich der im ersten und zweiten Vierteljahr hervorgetretenen Verschlechterungen schließen. Hinsichtlich der Gewichtsabnahme vor und während des Krieges ist zu bemerken, daß diese sich mit zunehmendem Alter vergrößert und vom 14. bis 17. Lebensjahre, also gerade während der Pubertät, ihr Maximum erreicht. Der Prozentsatz der als mager zu bezeichnenden Kinder belief sich im Sommer 1916 auf 10-18, während er im Jahre 1916 nur 7-15 batrug. Eine Zunahme der Zahl blutarmer Kinder und ein Nachlassen derWiderstandskraft derselben gegen Infektionskrankheiten ließ sich während des Krieges nicht konstatieren. Wenn auch die festgestellten Gewichtsverluste und ein gewisser Mangel des Fettansatzes Beachtung verdienen, so liegt doch, meint Schlesinger, ein Anlaß zu Besorgnis in betreff der Ernährung nicht vor. - J. Lehf eld t t $^{4}$ hat den Kräfte- und Ernährungszustand der seiner schulärztlichen Aufsicht unterstellten Volksschulkinder Magdeburgs trotz der während des Krieges verminderten Kost durchaus günstig gefunden. Auch die übrigen Magdeburger Schulärzte, mit Ausnahme von zwei derselben, haben, wie aus ihren Berichten hervorgeht, keine Veränderungen im Gesundheitszustand zum Schlechteren gegen die Vorjahre feststellen können. Selbst wenn der Krieg noch sehr lange dauert, können wir, meint Lehf eld t, um die Ernährung unserer Schulkinder vollständig unbesorgt sein. Lehfeldt führt noch das der Statistik entnommene Zahlenmaterial über die Sterblichkeit von Säuglingen und größeren Kindern an, woraus sich ergibt, $\mathrm{da} \beta$ diese seit 1911 abgenommen hat. - Nach Beobachtungen des Schularztes O. K ürbs in Eisenach ${ }^{5}$ ) sind Ernährungs- und Stoffwechselkrankheiten im Sommerhalbjahr 1916 nicht zahlreicher als in früheren Jahren aufgetreten. Kürbs erblickt darin einen Beweis, daß die heutige Ernährungsweise dem an passungsfähigen Organismus der Kinder bis zum sechsten Lebensjahre zusagt und wahrscheinlich auch nicht so erheblich von der früheren Ernährungsweise abweicht, als das bei Erwachsenen der Fall ist. - Der Stadtarzt K. Oschmann ${ }^{6}$ ) hat während der Jahre 1913 bis 1916 mit Schülern und Schülerinnen in Weißenfels jedesmal zu Beginn des zweiten Quartals Messungen und Wägungen vorgenommen. Er fand, daß die Zunahme an Größe und Gewicht im Jahre 1915/1916 zwar geringer als in den Vorjahren war und auch der Gewichtsstillstand und die Gewichtsabnahme zuungunsten des letzten Jahres aus-

1) Trüpers Zschr. f. Kindforsoh. 1916 S. $248 .-{ }^{2}$ ) M. m. W. 1917 Nr. 242. - ${ }^{3}$ ) D. m. W. 1917 S. $95 .-{ }^{4}$ ) Zschr. f. Schulgesdhtspfl. 1917 S. 33. - 5) Zschr. f. Schulgesdr+spfl. 1917 S. 46. - 6) Zschr. f. Schulgesdhtspfl. 1917 S. 49. fielen, $d a ß$ aber der allgemeine Ernährungszustand trotzdem fast derselbe geblieben war. Diesen Befund schreibt er dem Umstande zu, daß der Eiweiß- und Kalorienverbrauch der Bevölkerung vor dem Kriege den physiologischen Bedarf erheblich überschritten hat und daß man mit der Hälfte der sonst als notwendig angesehenen Mengen ausreicht.

Anders liegen die Befunde bei Erwachsenen. Der bekannte Kliniker Fr. v. Müller in München hat nach Angabe der Münchener Neuesten Nachrichten und nach brieflicher Mitteilung mit etwa 500 erwachsenen Personen Wägungen vorgenommen. Das Ergebnis derselben war, daß die wohlhabendere Bevölkerung ungefähr $10 \%$, die ärmliche $3-4 \%$ an Gewicht während der Kriegszeit verlor. In kleineren Städten ist die Gewich tsabnahme geringer. Auf dem Lande hält sich das Körpergewicht ungefähr auf dem früheren Niveau. Alte Leute zeigen durchschnittlich eine größere Gewichtsabnahme als jüngere, große und fette Personen eine bedeutendere als magere. Gewichtsabnahmen von $25-35 \mathrm{~kg}$ (nicht Pfund) sind keine Seltenheit.

Obwohl die vorliegenden Untersuchungen an Jugendlichen der Hauptsache nach alle zu dem gleichen und sehr erfreulichen Ergebnis kommen, $\mathrm{da} B$ von einer Entwicklungsbeeinträchtigung infolge der durch die Kriegsverhältnisse bedingten Einschränkung der Kost nicht die Rede sein könne, und obwohl Schlesinger besonders betont, daß Gewichtsverminderungen auf einen bedeutungslosen Fettverlust zurückzuführen seien, lassen sich einige Einwände gegen die Untersuchungsmethodik doch nicht unterdrücken. Zunächst ist zu bemerken, daß es bei qualitativ und quantitativ verminderter Nahrung keineswegs nur zu einer Fettabnahme kommt, sondern daß der Organismus den Mangel in der Zufuhr von Nahrungsstoffen außer durch Einsehmelzung des Fettes auch noch durch den Verbrauch anderer Beservestoffe auszugleichen sucht. Dahin gehören einerseits Kohlehydrate, deren Anwesenheit in den Muskeln eine gewisse Arbeitsbereitschaft derselben gewährleistet, anderseits das Vorratseiweiß. Unzureichender Ersatz beider kann verhängnisvoll werden. Ferner ist darauf hinzuweisen, daß außer den Beobachtungen von Bowditsch, Crichton Browne, Erismann, Hertel, Key, Kotelmann, Pagliani, Pesskoff, Quetelet, Roberts, Vahl und Wreetlind über Längenwachstum und Gewicht während der verschiedenen Lebensjahre, insbesondere im Pubertätsalter, jahrelang fortgesetzte, täglich mehrmals wiederholte Messungen und Wägungen von $R$. Malling $-\mathrm{H}_{a} \mathrm{nsen}^{1}$ ) vorliegen, durch die nachgewiesen wird, daß die Jahreszeiten auf die körperliche Entwicklung der Kinder einen bedeutenden Einfluß ausüben. Malling - Ha nsen fand, daß auf eine Periode schwachen Längenwachstums, die von Ende November und Anfang Dezember bis Ende März und Mitte April reicht, ein allmählich sich verstärkendes Längenwachstum folgt, während Gewichtszunahme unterbleibt bzw. sich auf ein Minimum reduziert und sogar in Gewichtsabnahme übergeht. Diese zweite Periode dauert von März-April bis Juli-August. Darauf folgt eine dritte, bis in den November und Dezember sich erstreckende Periode, in der das Längenwachstum ein Minimum aufweist, das Gewicht jedoch stark zunimmt. Diese Vorgänge werden durch die Lage der Schulferien insofern modifiziert, als diese in der Regel eine Gewichtszunahme mit sich bringen, die sich mit der Ferienlänge erhöht. Später hat Schmidt-Monnard²) die Beobachtungen von Malling- $\mathrm{H}_{a}$ nsen durch Massenuntersucliungen an Kindern in Halle mit einigen Abänderungen bestätigt. Auch die Schlesing erschen Angaben über den nor malen Gang der Entwicklung decken sich zum Teil damit. Natürlich muß bei allen Messungen und Wägungen, durch die ein Einfluß der Kost auf Wachstum und Gewicht festgestellt werden soll, sowohl auf die regelmäßigen Entwicklungsverhältnisse während der einzelnen Lebensjahre als auch auf die durch die Jahreszeiten bedingten periodischen Schwankungen des Wachstums und Gewichts Rücksicht genommen werden, wenn sich nicht ungenaue Ergebnisse einstellen sollen. Obwohl nun einige deı schulärztlichen Untersuchungen an gleichaltrigem und gleichgeartetem Schülermaterial und auch zu denselben Jahreszeiten vor dem Kriege und in den Kriegsjahren vorgenommen wurden, können sie dennoch nicht als einwandfrei betrachtet werden, da die klimatischen Verhältnisse (Sonnenstrahlung, Temperatur und die übrigen Witterungsverhältnisse) am gleichen Orte und während der gleichen Zeit der in Betracht kommenden Jahre ganz erheblich voneinander abweichen, wodurch sich die Untersuchungsergebnisse nebst ihren graphischen Aufzeichnungen verschieden gestalten müssen.

$\mathrm{Zu}$ einem weiteren Einwand gegen die Zuverlässigkeit der Methodik gibt der Schulbetrieb Anlaß. Während der Kriegszeit konnte derselbe nicht immer in gleichem Umfange und mit der gleichen Intensität aufrechterhalten werden wie in früheren Jahren, sondern beide haben vielfach Veränderungen erfahren. Es ist nämlich seit Beginn des Krieges eine gewisse Entlastung der Schüler, ein Zuwachs an schulfreier Zeit und damit ein vermehrter Aufenthalt der Jugend im Freien ein-

1) Perioden im Gewicht der Kinder und in der Sonnenwärme, Verlag von Vilh. Tryde, Kopenhiagen 1886, darin auch frühere Arbeiten desselben Verfassers zitiert. $-{ }^{2}$ ) Zschr. f. Kindhlk. 1895 S. 84. 
getreten, wodurch auf die körperliche Entwicklung derselben zweifellos ein günstiger Einfluß ausgeübt wurde. Wenn daher nach Engelhorn nervöse Erscheinungen, Aengstlichkeit des Wesens, Störungen des Schlafes und andere psychische Verstimmungen während der Kriegszeit seltener und in geringerem Grade beobachtet werden als vor dem Kriege, so ist dies wohl weniger der vereinfachten Kost als der verminderten $\mathrm{Be}$. anspruchung der Jugend zuzuschreiben. Dies läßt sich sogar nach den Ausführungen Engelhorns bis in die Hilfsschule hinein verfolgen. Diese mußte an verschiedenen Orten aus Mangel an Lehrkräften geschlossen werden. Die Zöglinge wurden der Normalschule zugeteilt. $\mathrm{Da}$ hier aber die meisten von ihnen nicht mitkommen konnten, überließ man sie ,ihrem Schicksal“" und betrachtete die Schule für sie nur als Auf. bewahrungsort, man kann hinzufügen, als Erholungsanstalt. Solche und ähnliche Verhältnisse sind entschieden zu berücksichtigen, wenn es sich um anthropometrische Bestimmungen an Jugendlichen handelt. Thiele steht mit seinen Angaben über Zunahme des Wachstums und Gewichtes von Schülern während des Krieges zwar isoliert unter den übrigen Beobachtern, man darf die Ergebnisse jedoch deswegen nicht gleich als unwahrscheinlich betrachten. Ich frage aber: Wie müssen dann in Chemnitz die Schulverhältnisse gelegen haben, wenn die Schüler trotz beschränkter Kost so prachtvoll gedeihen konnten?

Oder war Chemnitz doch vielleicht reichlicher mit Nahrungsmitteln versorgt als Straßburg und andere Orte? -

Ein weiterer Einwand gegen die Methodik betrifft die Leistungsfähigkeit der Schüler. Wenn diese, insbesondere auf geistigem Gebiete, während der Kriegszeit wirklich unverändert geblieben ist, wie Schlesinger und andere meinen, so können hierzu ebenfalls Erleichterungen im Schulbetriebe beigetragen haben. $\mathrm{Ob}$ dieser Fall eingetreten ist, ließe sich durch Vergleich der Themata für die schriftlichen Arbeiten sowie der Beschaffenheit und des Umfanges des im mündlichen Unterricht behandelten Stoffes während des Krieges und vor diesem ermitteln. Durch Vergleich der Unterrichtsergebnisse würde man zu einem Urteil über die Leistungsfähigkeit gelangen können, vorausgesetzt, daß die Anforderungen zu beiden Zeiten die gleichen waren. Unter Berücksichtigung der 1916 eingeführten Sommerzeit, wobei der Nachmittagsunterricht in die heißeste Tageszeit fällt, müßte auf die Ermüdbarkeit während des Unterrichts und bei Anfertigung der häuslichen Schulaufgaben Bezug genommen werden; denn auf die Ermüdbarkeit ist die Kost nicht ohne Einfluß. Auch die körperlichen Leistungen dürfen bei Untersuchungen über die Kriegskostwirkung auf den Ernährungszustand nicht außer acht gelassen werden. $-\mathrm{Daß}$ bei qualitativ und quantitativ verminderter Nahrung Beschaffenheit und Funktion der Gewebe und Organe Beeinträchtigung erfahren können, ist bekannt. Wie und wo diese erfolgt, ist, außer bei Schwund des Pannikulus, also insbesondere in inneren Organen, manchmal nur schwer zu ermitteln. Sie kann sich aber auch in auffälliger Weise offenbaren. Dietrichs ${ }^{1}$ ), B. Schweitzer ${ }^{2}$ ) und M. Graef $\mathrm{e}^{3}$ ) berichten über ein in der letzten Zeit sich häufendes Aussetzen der Menstruation (Kriegsamenorrhoe) nichtschwangerer Mädchen und Frauen als Folge der veränderten und verminderten Ernährung. Es handelt sich dabei um eine Störung der inneren Sekretion der Eierstöcke, bei der, falls sie dauernd wird, mit der Möglichkeit fortschreitender Atrophie des Uterus und mit Fortpflanzungsunfähigkeit zu rechnen ist. Auch Uterusverlagerung und Scheidenvorfall werden infolge hochgradiger Abmagerung bei der Kriegskost beobachtet. -

Da man durch Wägungen ebenso wie durch eine auf die Straffheit der Haut und Muskeln, die Farbe und Blutfülle der Schleimhäute sowie den Gesamthabitus gerichtete Inspektion, die oft mit viel Subjektivität verbunden ist, den Ernährungszustand nur annähernd abzuschätzen vermag, so bedürfen die bisherigen Untersuchungen über diesen, sofern sie wissenschaftlichen Wert haben sollen, der Ergänzung und Erweiterung. Um diese zu ermöglichen, sind experimentelle Prüfung der Leistungsfähigkeit und Ermüdbarkeit (ergographische, dynamometrische und andere Methoden) sowie Stoffwechseluntersuchungen unentbehrlich. Meines Wissens sind erstere bisher nicht, letztere nur sehr spärlich an Schulkindern ausgeführt worden. Es hat aber R. Rosenfeld (Breslau) ${ }^{4}$ ) an sich und einer zweiten Versuchsperson mittels des Ergographen über den Einfluß von Kostveränderungen auf die Muskelleistung Beobachtungen angestellt, wobei sich ergab, daß eine vorzugsweise laktovegetarische Kost im Gegensatz zur Fleischkost seine Muskelleistung nicht unerheblich herabsetzte, während dies bei der zweiten Versuchsperson nicht der Fall war. Demnach scheint auch individuelle Disposition bei der Ernährung eine Rolle zu spielen. L. Licht. witz $z^{5}$. hat an Bürgern, darunter auch 19 Kinder wohlhabenderer Eltern im Alter von 2 bis 14 Jahren und 21 Volksschulkinder im Alter von 6 bis 14 Jahren, sowie an Kriegsgefangenen in Göttingen Stoffwechseluntersuchungen vorgenommen. Die 24stündige Harnmenge möglichst

1) Zbl. f. Gyn. 1917 S. 157. - ") M. m. W. 1917 S. 551. 3) M. m. W. 1917 S. $579 .-{ }^{4}$ ) Zschr. f. physik. u. diät. Ther. 1916 S. 97. - 5) B. klin. W. 1916 Nr. 34 u. 41. vieler Menschen wurde auf Stickstoff analysiert und daraus der Eiweiß. gehalt und Brennwert der Nahrung berechnet., Er fand, daß diese zwar nicht als reichlich, aber doch als ausreichend betrachtet werden konnte. Die Untersuchungen sind, insbesondere wegen der Schwierigkeit der Beschaffung der gesamten 24stündigen Harnmenge und der Entscheidung der Frage, ob die Kartenkost streng eingehalten wurde, mit Vorsicht aufzunehmen, für die soziale Bewertung aber doch insofern brauchbar, als sie die Lage nicht besser erscheinen lassen, als sie in Wirklichkeit ist. Fr. v. Müller fand im Verlaufe seiner genannten Beobachtungen bei strenger Einhaltung der Kartenkost ein Stickstoffdefizit bis zu $1 \mathrm{~g}$. -

Dem experimentellen Studium des Einflusses der Qualität und Quantität der Kost auf den Ernährungszustand, namentlich bei der durch den Krieg bedingten Lebensmittelknappheit, steht noch ein weites Feld offen. Die durch Tierversuche H. Arons (Biochem. Zschr. 1911; 30, S. 207 u. B. kl. W. 1914 Nr. 21) bekannt gewordene Tatsache, $\mathrm{da} ß$ Wachstum ohne entsprechende Gewichtszunahme möglich ist und daß dieses Mißverhältnis manchmal eintritt, wenn eine Nahrung, die in genügender Menge normale Verhältnisse bewirkt, in unzureichender Menge gereicht wird, erfordert insofern Beachtung, als dadurch die Periodizität beeinflußt werden kann, Besonders wichtig sind die richtige Bewertung der periodischen Körperwachstums- und Körpergewichtsschwankungen in ihren Beziehungen zur Art und Menge des Nahrungsmaterials, die Ermittlung des Optimums des zur Erhaltung des Stickstoffgleichgewichts erforderlichen Kostmaßes und die Erforschung des Ernährungsbedürfnisses der einzelnen Organe unter Berücksichtigung ihres Anpassungsvermögens sowie etwa erforderlicher Funktionszulagen für diese, je nach ihrer Flächen- und Volumenentfaltung und ihrer Beanspruchung auf Leistungsfähigkeit einschließlich extremer Fälle bei geistiger und körperlicher Betätigung des Individuums. $\mathrm{Zu}$ berücksichtigen sind für die Beurteilung des Nahrungsbedürfnisses auch noch die durch v. Pirquet (Zschr. f. Kindhlk. 1916 Bd. 14 H. 3; 1917 Bd. 15 H. 3/4 u. 5/6) gekennzeichneten Beziehungen, die zwischen der Darmfläche, der Sitzhöhe und dem Körpergewicht bestehen. - Je umfassender und genauer die Untersuchungen ausgeführt werden, desto besser und leichter wird sich die Verabreichung einer rationellen Kost ermöglichen lassen zum Segen der Jugend und des ges:mten Volkes. 